All those debriefing have been trained in the correct techniques to ensure the participants and observers are allowed to reveal the learning points and sessions are all videoed and used during the debrief as appropriate, to emphasize particularly areas for development or highlight good practice.

Results Retrieval team members were initially apprehensive of this new approach to retrieval training but have found it useful giving positive feedback and encouraging colleagues to attend.

Conclusions We will continue to use this approach to provide well prepared teams who are clinically competent and aware of the human factors in every retrieval situation.

\section{POST-TRAUMATIC STRESS DISORDER AFTER DISCHARGE IN PEDIATRIC INTENSIVE CARE UNIT (PICU)}

doi:10.1136/archdischild-2012-302724.1907

D Sönmez Düzkaya. Istanbul University, Istanbul Faculty of Medicine Directorate of Nursing Services, Supervisor Nurse, Istanbul, Turkey

Post-Traumatic Stress Disorder (PTSD) develops after exposure to an extremely traumatic event such as death, injury, or physical threat to self, family member, or other significant person.

Admission to a paediatric intensive care unit (PICU) with acute disease carries a high level of stress for families, as the disease is of sudden onset and is life-threatening. The incidence of PTSD (21\%) was significant among parents well after their child was discharged from the PICU.

Symptoms of PTSD include

a. reexperiencing the traumatic event through recurrent, intrusive recollections, repetitive play, or distressing dreams;

b. avoidance of associations with the event through restricted affect and feelings of detachment; and

c. hyperarousal symptoms such as sleep difficulties, poor concentration, irritability, aggression, and physiological reactivity to trauma-related events.

In addition to general hyperarousal symptoms, children may complain of stomachaches and headaches.

There have been many summaries and reviews of research published regarding the effects of hospitalization with these children. Findings indicate that the experience is, indeed, stressful and that children may experience fear phenomena, regression, sadness, separation anxiety, withdrawal, sleep disturbances, and aggressive behaviors. There was a positive correlation between PTSD symptoms in parents and

1. PTSD symptoms in the child

2. length of admission and

3. perceived threat of illness to the child's life.

In PICU admissions cause greater disruption to a child's life, with longer admissions, greater time off school and more paediatric out-patient contacts and hospital re-admissions in the 6-12 months after discharge.

\section{MANAGEMENT OF PULMONARY HYPERTENSION AFTER PAEDIATRIC CARDIAC SURGERY}

doi:10.1136/archdischild-2012-302724.1908

'GJ Wildeboer, ${ }^{2} \mathrm{E}$ Ponne, ${ }^{2} \mathrm{H}$ Geerts. ${ }^{1}$ Kinder IC (CA 59); ${ }^{2} U M C G$, Groningen, The Netherlands

Introduction Treatment of pulmonary hypertension in children has significantly improved over the years. The Beatrix Children's Hospital serves as the nationwide referral center for these children. Of importance, pulmonary hypertension occurs in a considerable proportion of patients after cardiac surgery. Our paediatric intensive care unit admits approximately 180 patients annually after cardiac surgery. About $5 \%$ of these children develop pulmonary hypertension. Its occurrence may significantly affect the postoperative disease course during the first 72 hours of PICU stay.

Aim The provide insight into early recognition and management of pulmonary hypertension after cardiac surgery.

Methods We have developed nursing protocols describing how to monitor and interpret haemodynamic parameters, and how to interpret laboratory and roentgenologic investigations. Special attention is paid towards the clinical appearance of the patient. Next to this, supportive tools such as nitric oxide and highfrequency oscillatory ventilation (HFOV) are inevitable.

Results A protocolized approach allows us to recognize complications after paediatric cardiac surgery early during PICU stay. As a consequence, early management is possible.

Nitric oxide and HFOV are used as a supportive intervention for managing pulmonary hypertension.

Conclusion A protocolized approach allows us to recognize complications after paediatric cardiac surgery early during PICU stay.

\section{THE EFFECTS OF TEACHING ON THE KNOWLEDGE OF MOTHER TO CARING FOR INFANT \& CHILDREN AT HOME, FOLLOWING CARDIAC SURGERY}

doi:10.1136/archdischild-2012-302724.1909

S Montaseri, E Marvasti, MA Navabi, GH Ajami, A Ayatollahi. Shiraz University of Medical Sciences, Shiraz, Iran

Congenital heart diseses, are chronic illness in infants \& children. This research is a quasi-exprimental study investigating the impact of education on home care knowledge of sixty mothers with children undergoing heart surgery. Data collection consisted of a pretest, posttest and retentional questionnaire that contain of demographic data of mother \& her baby and multiple choice test relate to heart diseases, surgical treatment, complication and home care of babies. The mothers gained a positive mark for true answer, a negative mark for a false answer and a zero mark for an unknown answer. Data collection was conducted in before admission of babies, one week and one month after discharge. As for the case group teaching programs consisted of educational vido films and a face to face teaching and educational booklet. For the data analysis a spss package \& descriptive statistic were employed. To identify the level of mother's knowledge, mother's mark were classified as, good-moderate and weak. The result obtained showed that at pretest. the subjects in the case group did not have a good level. As for the control group only $6.7 \%$ were evaluated as good level at pretest. At posttest and retentional phases scors increased to 100 and $96.7 \%$ respectively for the case group. Regarding the control group $6.7 \%$ were evaluated as good and dropped to zero at the retentional stage. However, comparsion of the mean score at the posttest and retentional in both groups, showed a a decrease of knowledge overtime. In conclusion the results of the present study significantly supports the impact of education on improving the knowledge of mothers on providing a better home care for children undergoing heart surgery.

\section{NURSING MANAGEMENT AND CARE OF THE CHILDREN ON MECHANICAL CIRCULATORY SUPPORT}

doi:10.1136/archdischild-2012-302724.1910

F Petrangeli, L Del Sere, L Luciani, P Cogo. Department of Pediatric Cardiology and Cardiac Surgery, Bambino Gesù Children's Hospital, IRCCS, Rome, Italy

Background and Aims The number of children being hospitalized for heart failure (HF) is increasing and, in recent years, mechanical circulatory support (MCS) is gaining more importance in the treatment end stage HF. The use of these complex technology implies changes in nursing assistance with equally complex aspects of care. The aim of this work was to describe our experience in the 